dimensions - the four dimensions of relativistic space-time and six others of which we are unaware. Thus there is room for ample orthogonality to generate selection rules that prevent bizarre happenings between particles.

The most persuasive part of Greene's excellent book is that in which he persuades the reader that the problem of the six hidden dimensions is not a problem but a matter of perspective. A garden hose seen from a great distance looks like a one-dimensional object, but close up it is plainly a two-dimensional surface on which motion perpendicular to the length is perforce circular and repetitive. The length of the hose is Greene's analogy for an ordinary extended dimension; the perpendicular circular tracks stand for one of the six wrapped-up dimensions. If the radius of the circle (or of the garden hose) is small enough, it is recognizable only in close-up (which means at the highest energy).

How small are the compact radii? It seems to be agreed that the compact dimensions must be curled up with a radius in the neighbourhood of the Planck length, which is the constant with the dimensions of length formed from Planck's constant, Newton's gravitational constant and the velocity of light. Formally, the length is $\mathrm{V}\left(h G / c^{3}\right)$, where $h$ and $G$ are the quantum and gravitational constants and $c$ is the velocity of light. Numerically, the length works out at $10^{-35} \mathrm{~m}$, which is small enough for Greene's purposes.

This is the kindergarten stuff of string theory, but Greene shrinks from no obstacle in the path of understanding, instead turning each into an opportunity to make the field exciting. The doctrine of supersymmetry has become an intrinsic part of string theory - otherwise 'superstring' theory. The implication is that there are as many fundamental particles yet to be discovered as are now known, but all of them are much more massive. String theory will fall if it is ever shown that they do not exist.

The essential step forward, and the cause of Greene's rekindled enthusiasm, was taken by Witten in 1995. He showed that the five alternative string theories then defined are essentially equivalent. A weak coupling constant (or the strings' equivalent of electric charge) in one will be the equivalent of another theory with a strong coupling constant. So, says Greene, the road points ahead to the age-old dream of predicting both the contents of the Universe and the properties of those contents. Already there are people working on the notion that black holes are merely very massive elementary particles.

Even if the dream proves false, Greene has brought an absorbing field of enquiry to vivid life. The supposition that particles are not points but strings fits well with familiar phenomena. The fact that all particles of matter have intrinsic spin (which may be zero) has always provoked the question of whether spin is a property of particles or of space. The materialization of particles from apparently empty space is similarly provoking. String theory neatly answers them all.

So what lies ahead? Not even Greene is sure. String theory may not turn out to be the cat's whiskers he hopes. There are alternatives, such as Roger Penrose's twistor theory (which Greene reckons may say the same as strings). The most imaginative suggestion in this imaginative book is that the time has come to solve problems of quantum gravity in strictly quantum language and not by posing them in classical terms and then 'quantizing' them. Meanwhile, there is a whole raft of algebraic geometry to be done. The thousand and more people working in the field will need courage to do these largely thankless chores, but this splendid book will cheer them on their way.

Sir John Maddox is at 9 Pitt Street, London W8 4NX, UK.

\section{The self-centred meme}

\section{The Meme Machine}

by Susan Blackmore

Oxford University Press: 1999. 258 pp.

$£ 18.99, \$ 25$

\section{Jerry A. Coyne}

The meme, defined as "any unit of cultural transmission or any unit of imitation", was introduced by Richard Dawkins in the final chapter of The Selfish Gene. Dawkins' message was that any entity with the gene-like

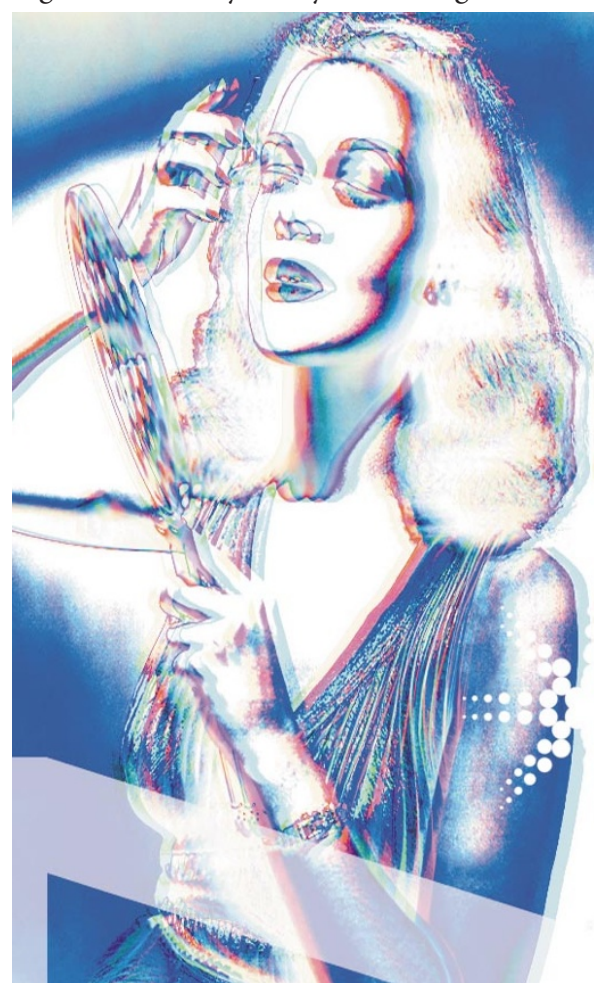

ผ๑ 1999 Macmillan Magazines Ltd properties of replication, variation and competition is a "selfish replicator" that can spread through populations by an analogue of natural selection. Memes qualify as replicators because bits of culture can be copied by imitation and compete with other units for human attention. Thus, despite the differences between memes and genes (genes, for example, are almost never passed to unrelated individuals), those memes most easily replicated and mimicked could proliferate, causing swift and important cultural change. Alarmed by the rampant and uncritical adoption of this analogy by philosophers, psychologists and the popular press, Dawkins downplayed the importance of memes in his later works.

However, in his foreword to Susan Blackmore's The Meme Machine, Dawkins notes that: "I was always open to the possibility that the meme might one day be developed into a proper hypothesis of the human mind, and I did not know how ambitious such a thesis might turn out to be." The thesis, called "memetics", has now arrived, and it is indeed ambitious. Unfortunately, Blackmore's book, aimed at both general readers and academics, proves to be a work not of science, but of extreme advocacy. Teeming with untestable speculations, indifferent to alternative theories and almost too grandiose to be taken seriously, The Meme Machine offers a convoluted and wholly unsatisfying - explanation of cultural and biological evolution.

Blackmore defines memes as "units of imitation"; her examples from humans include songs, fax machines, books, alcohol, and any device, behaviour or idea that can be copied. Memes can also be combined into

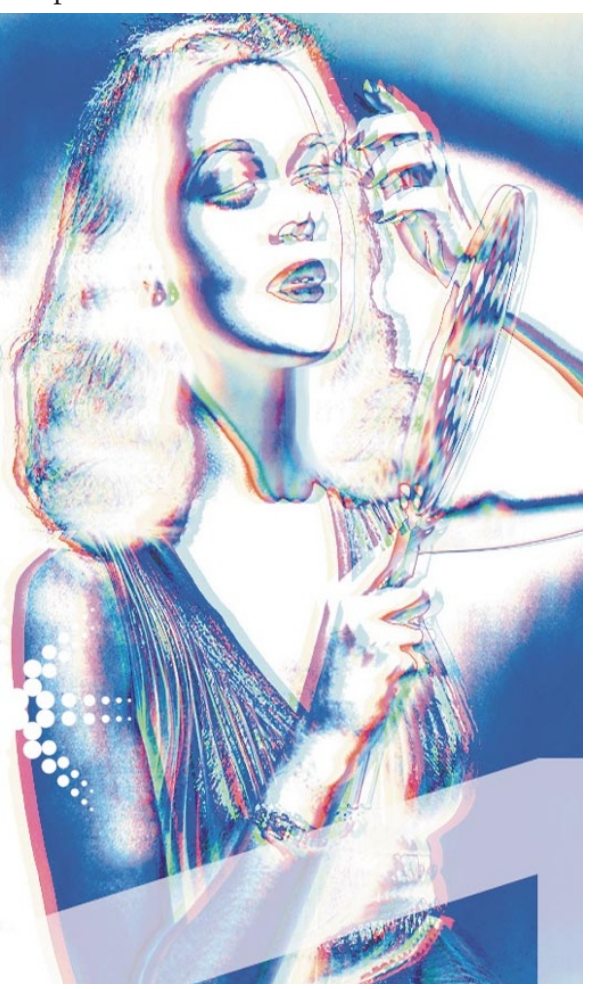


"co-adapted meme complexes" that include a stimulus for imitation ("good girls don't have sex before marriage"), and into even larger "memeplexes", such as religion, with many copy-able elements. Like Sherlock Holmes, Blackmore sees our brain as a room of limited size that can store only so many memes. A meme's success depends not on its benefits to the carrier, but on its ability to be replicated, retained and imitated. As she declares persistently: "If a meme can get itself successfully copied it will." According to Blackmore, this shibboleth explains major aspects of our biology and culture, including oversized brains, language, technology, writing, contraception, adoption, altruism, vegetarianism, New Age ideas such as alien abduction, and consciousness.

Such an ambitious theory demands close scrutiny, but space restricts me to exploring only a few of Blackmore's views. She suggests, for example, that enlargement of the human brain began when natural selection improved the ability to imitate, followed by selection for ever more neurons to store successful memes. Blackmore contends that such selection has occurred only in hominids and birds that learn songs. Subsequently, memes further expanded our brain through sexual selection: as better imitators, bigger-brained individuals are more attractive mates and leave more offspring.

One immediately smells trouble. First, there is no evidence that brain-size increase had anything to do with memes - there are as many explanations (including language, social grouping, hunting) as there are evolutionists, and no way to judge which theory is best. Indeed, the meme hypothesis seems among the least likely. As others have noted, the serious proliferation of memes began roughly 30,000 years ago when humans commenced their march to larger social groups, writing and complex culture. The human brain, however, stopped enlarging after reaching its present volume nearly 500,000 years ago. Why did brain-size evolution stop so long before the heaviest rain of memes?

Although Blackmore deems memetics a scientific idea, nearly all of her suggested tests are either impossible to perform or unable to rule out competing theories. For example, she claims support for the sexual selection of memes, declaring that "... being highly articulate makes you sexually attractive. The history of love poems and love songs suggests as much, as does the sexual behaviour of politicians, writers, and television stars." This may be true, but Blackmore has forgotten sports heroes, rock stars and supermodels, also highly attractive but hardly known for eloquence. The combined data better

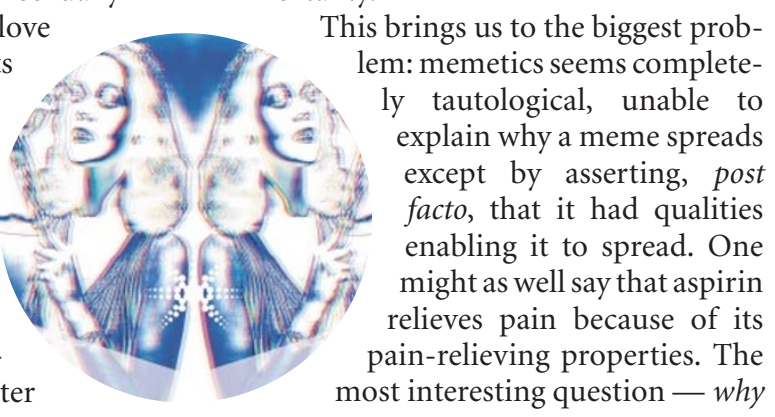
m: memetics seems completetautological, unable to explain why a meme spreads except by asserting, post facto, that it had qualities enabling it to spread. One might as well say that aspirin relieves pain because of its most interesting question - why support the sociobiological theory that humans (especially females) are attracted by power and its attendant resources.

Narrower hypotheses have additional difficulties. A frequent problem is that the supposed meme seems to discourage its own propagation. Such a case is Blackmore's view that celibacy in Catholic priests is a meme that spreads by forcing clergy to divert their sexual energy into serving their religion. But what memes spring from this suppressed sperm? If priests beseech their flocks to forever refrain from reproduction, it is news to me. Other religions with non-celibate clerics, such as Islam, seem to have no trouble attracting believers.

Finally, Blackmore sees consciousness as a "selfplex", an insidious band of memes that conspire to give their carrier a false sense of self. Consciousness permits personal conviction, said to be especially good for encouraging imitation (for example, "I love beer"). However, consciousness cannot be illusory: as the American philospher John Searle has argued, thinking one is conscious is identical to being conscious. Moreover, Blackmore's scenario denies the possibility of consciousness to meme-less animals, and fails to explain the hardest problem of human consciousness: subjective sensation. How can memes account for the pain I feel when I pinch myself?

But this is mere quibbling, for Blackmore's enterprise has two fatal flaws. First, she has got the chain of causation backwards. The claim that memes created major features of humanity is equivalent to the claim that the main force driving the development of better computers has been the self-propagation of software. In reality, computers are usually designed for speed and capacity, which then permits the development of new software. Similarly, the selfreplication of memes does not mould our biology and culture; rather, our biology and culture determine which memes are created and spread. What a world of human psychology is obscured by Blackmore's mantra, "If a meme can get itself successfully copied it will"! To me, memetics boils down to the following obvious theory: ideas tend to spread if they cater to our desires to have love, comfort, pleasure, power, sex, the attention and admiration of others, a meaningful life and a way to evade the awful fact of mortality.

This brings us to the biggest prob- some memes spread and not others - is completely neglected. Why did Christianity take hold during the waning days of the Roman Empire? You won't find the answer, or any way to attain it, in memetics. (This, by the way, makes memetics utterly unlike biological evolution. The spread of genes through natural selection is not tautological because one can predict their fate through their known effects on replication and the reproduction of their carriers.)

In a final effort to propagate her ideas, Blackmore constructs an extremely clever coadapted meme complex: "Evolutionary theory faced enormous opposition because it provided a view of humans that many humans do not like. The same will probably be true of memetics." Well, call me Bishop Wilberforce, but in my view memetics is but a flashy new wrapping around a parcel of old and conventional ideas.

Jerry A. Coyne is in the Department of Ecology and Evolution, The University of Chicago, 1101 East 57 Street, Chicago, Illinois 60637, USA.

\section{Shady secrets of the Enlightenment}

\section{Sex and the Gender Revolution, Volume 1: Heterosexuality and the Third Gender in Enlightenment London}

by Randolph Trumbach

University of Chicago Press: 1998. 484 pp. $\$ 35, £ 27.95$

\section{W.F.Bynum}

Sex and bodies have become fashionable historical topics. Indeed, there are those who believe that historians are a prurient lot, preferring the bedroom to the laboratory, hospital, battlefield, palace and other sites where activities traditionally deemed more worthy of historical analysis take place.

Prurient or not, historians and social scientists quite correctly insist that bedroom behaviour can be exceptionally difficult to analyse. One of Randolph Trumbach's adulterers stuffed a handkerchief in the keyhole of her bedroom door, so that her servants could not spy on her. Even his randy men, who went with prostitutes in parks, alehouses or dark alleyways, were unlikely to leave detailed accounts of their escapades. This, of course, makes eighteenth-century diarists such as James Boswell and William Byrd so attractive to historians, even if their class and status make them unsuitable for generalization about male behaviour down the ranks.

For the most part, then, Trumbach's account of heterosexuality in eighteenthcentury London relies on other kinds of evidence, including newspapers, diaries, correspondence and imaginative literature. 